\title{
Mechanical Modeling of Bend Sensors Exploited to Measure Human Joint Movements
}

\author{
Giovanni Saggio $^{(\mathrm{a})(\mathrm{c}),}$ Paolo Bisegna ${ }^{\text {(b) }}$, Giuseppe Latessa ${ }^{(a)}$, Stefano Bocchetti ${ }^{\text {(a) }}$ \\ Dept. of (a) Electronic and (b) Civil Engineering, "Tor Vergata" University, 00133 Rome, Italy \\ (c) Centro di Biomedicina Spaziale, Via della Ricerca Scientifica snc, 00133 Rome, Italy \\ e-mail: saggio@uniroma2.it
}

\begin{abstract}
Flexibility, lightness, wearability and cheapness are the most important features for a successful adoption of bend sensors, being they fundamental elements to realize systems able to convert static positions and movements into electrical signals. Even though these sensors have been employed for many different applications, the focal aspect of their mechanical modeling is still not adequately considered. For such a reason the aim of this paper is to fill a lack concerning the method of bend sensors mechanical characterization and modeling. The results have been exploited to realize an instrumented glove able to measure finger joints movements.
\end{abstract}

Index Terms: Sensor modeling; mechanical modeling; wearable sensors; non implantable sensors

\section{Introduction}

Revealing and recording fingers movements is essential and helpful in many circumstances as medical applications, handicap, remote control, virtual simulations, music, support for athletes' training, etc. Fingers joints position and movements can be measured by means of the so called "instrumented glove", a definition referring to the ensemble of a support consisting of an elastic glove, transducers for mechanical-electrical data conversion and the conditioning electronics.

The transduction can be realized employing different technologies as flexible tubes light source, LED, fiber optic, electrical contacts, inductive length encoders, accelerometers, switches, gyroscopes, or sensors based on pressure, capacitance, electro- magnetic- opticeffects [1]. Here we report a system based on bend sensors which avails itself of their lightness and cheapness and permits to realize a comfortable, wearable glove.
Bend sensors [2] and piezoresistive sensors [3, 4] have been already used for the previously mentioned applications and their properties have been analyzed. Nevertheless no particular attention was paid to their mechanical characterization, so we added some improvements in order to fill this gap. We introduced a new measurement set-up and developed a mechanical model for the sensors useful to justify the results of the measurements and to furnish an important tool to predict sensors behavior in analogue circumstances.

\section{Instrumented glove}

A lightweight lycra-based glove was chosen for our instrumented system as a support for ink carbon based bend sensors. The sensors were placed onto the glove in correspondence to the dorsal part of all the fingers joints, in order to trace fingers movements, as illustrated in figure 1.

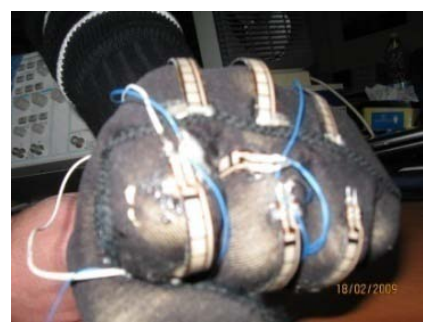

(a)

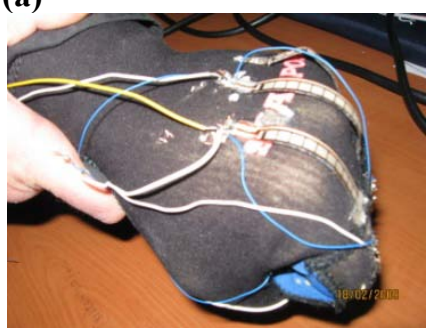

(b)

Figure 1. (a) Front and (b) lateral view of the sensors mounted onto the glove 


\section{Measurement Set-up}

A proper home-made measurement set-up was realized for the sensors characterization.

The set-up was mounted on a shock absorber bench to assure adequate stability. A stepper motor provided the rotation movements of an hinge made of anodized aluminum, so to furnish the necessary electrical insulation. One wing of the hinge was fixed to the central axis, the other one rotates with the motor, with a transmission ratio set to 1:1 (figure 2).

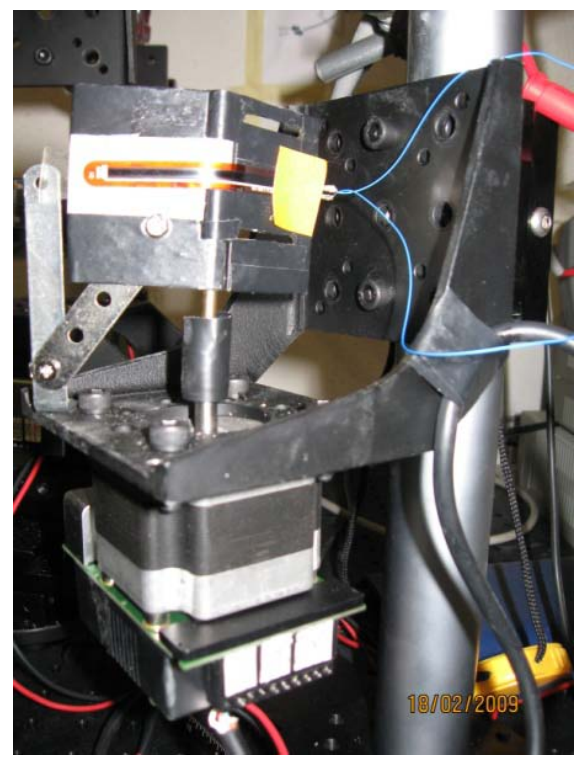

Figure 2. Hinge mounted on the stepper motor axis. One wing of the hinge was fixed to the central axis, the other one rotates with the motor thanks to a rigid mechanical joint.

The sensor used during the test was mounted on the dorsal part of the hinge, perpendicularly to the motor axis. The sensor tip was mechanically fastened to the fixed wing of the hinge, while the sensor bottom was held down by a sliding buttonhole. In such a manner a sensor can freely slide in and out while flexing. The buttonhole is a key point for accurate measurements, because it must allow the sensor to move without unwanted friction, avoiding at the same time the formation of a spatial gap with the hinge.

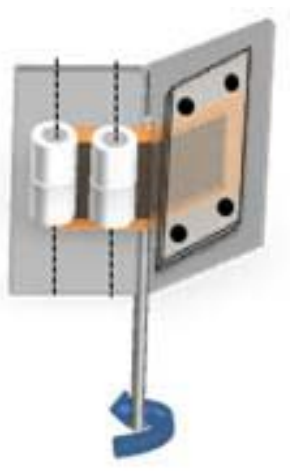

Figure 3. Sensor mounted on the hinge wings. The buttonhole is a key point for accurate measurements. Here it is shown an efficient bearing scrolling roll solution maintained by an outlying pivot

The overall setup was remotely controlled by a personal computer with a LABVIEW interface to automate the measurement procedure. The resistive response of the sensor was read by a 5.5 digits $34405 \mathrm{~A}$ Agilent digital multimeter.

\section{Sensor characterization}

Thanks to our set-up, we characterized two commercially available bend sensors, provided by Image SI and Flexpoint Inc. The sensors were in turn placed on the hinge body and the motor automatically moved one hinge wing from $0^{\circ}$ to $90^{\circ}$, stepped $10^{\circ}$. The most significant results obtained are reported in fig. 4.

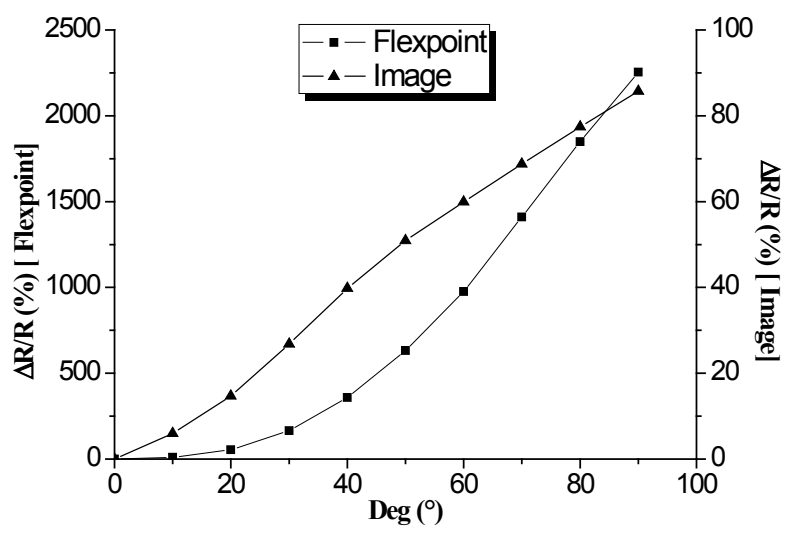

Figure 4. A comparison between two different bend sensors: relative resistance vs bending angle

In order to justify the results we propose here a mechanical model for sensors. 


\section{Mechanical model}

The strain sensitivity of a piezoresistor is indicated by its gauge factor (GF), which is defined as the fractional change in resistance $R$ due to an applied strain $\varepsilon$, i.e. $G F=\varepsilon \frac{d R}{R}$. There are various gauge factors and they depend on the direction of the applied strain $\varepsilon$. In this work, we consider the longitudinal gauge factor $G F_{L}$, which is relevant for the change in resistance when a strain $\varepsilon$ is applied across the length of the resistor, in parallel to the current flow. It is known to depend on the piezoresistivity coefficient of the film and on the mechanical properties of the carrier substrate, as shown by Papakostas and White [5].

In the following analysis it is assumed that the film is very thin compared to the substrate (so that it does not affect the stresses in the substrate) and conforms to the strains of the substrate in planar directions. Moreover, it is assumed that the substrate remains stuck to the hinge.

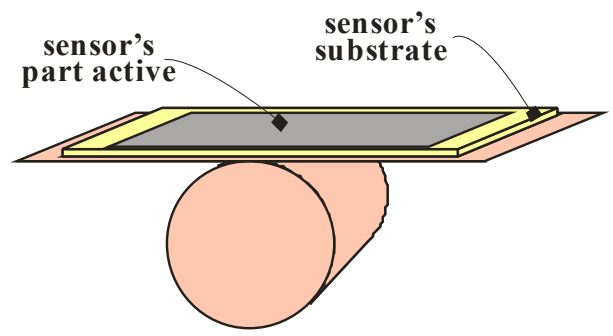

(a)

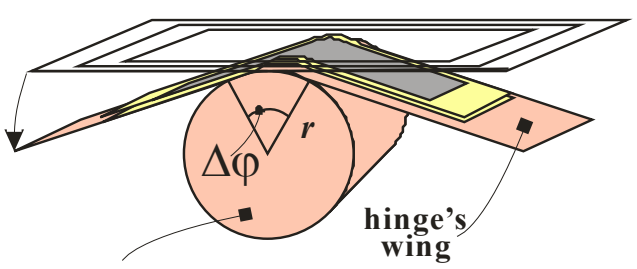

central axis

(b)

Figure 5. Sensor lying on the hinge wings in (a) flat and (b) angled position

Let $\Delta \varphi$ be the rotation of one wing of the hinge with respect to the other one. As depicted in figure 5, a portion of the piezoelectric sensor is bent on the hinge pin. The sensor can slip over the hinge, so that reference is made to the neutral plane of the substrate, which do not undergo strains. It is located on the middle of the substrate, and hence its distance from the pin centre is $r+h / 2$, where $r$ denotes the pin radius and $h$ is the thickness of the substrate. As a consequence, the length of the bent portion of the substrate is:

$$
l_{b}=\left(r+\frac{h}{2}\right) \Delta \varphi
$$

and its curvature is:

$$
\chi=\frac{1}{r+h / 2}
$$

The piezoresistor film bonded to the substrate turns out to be strained by the substrate bending over the same span length $l_{b}$, and the induced longitudinal strain is:

$$
\varepsilon=\frac{h}{2} \chi
$$

The relevant fractional change in resistance is:

$$
\frac{\Delta R_{b}}{R_{b}}=G F_{L} \varepsilon
$$

where $G F_{L}$ is the longitudinal gauge factor, and $R_{b}$ is the unstrained resistance of the span length $l_{b}$. The latter is a fraction of the unstrained resistance $R$ measured between the electrodes, enclosing a length $l$ of the piezoresistor film:

$$
R_{b}=\frac{l_{b}}{l} R
$$

$\Delta R_{b}$ coincides with the total resistance increase $\Delta R$, since the remaining portions of the film remains unstrained, and by the previous equations it easily follows that:

$$
\frac{\Delta R}{R}=\frac{R_{b}}{R} \frac{\Delta R_{b}}{R_{b}}=\frac{l_{b}}{l} G F_{L} \varepsilon=G F_{L} \frac{h}{2 l} \Delta \varphi
$$

This important relationship shows that the output signal $\frac{\Delta R}{R}$ is expected to be independent of the pin radius $r$, and to be linearly proportional to the input signal $\Delta \varphi$. Moreover, the sensitivity of the transducer is directly proportional to the longitudinal gauge factor $G F_{L}$ and to the substrate thickness $h$, whereas it is inversely proportional to the distance $l$ between the electrodes. Of course, according to the present analysis, the latter quantity cannot be inferior to the maximum value attained by $l_{b}$, which is given by the maximum rotation angle $\Delta \varphi$ times $r+h / 2$. 


\section{Discussion and Conclusion}

As a consequence of the previously developed mechanical model, we expected a linear behavior of the sensor resistive variation vs bending angles. Referring to figure 4 , this behavior is sufficiently exhibited by the Image sensors but the Flexpoint sensors demonstrated a behavior near linearity only after round $40^{\circ}$ and some degree of non linearity between $0^{\circ}-40^{\circ}$. It must be noticed that the thickness of sensors substrates is different: $0.2 \mathrm{~mm}$ for Flexpoint sensors and $0.5 \mathrm{~mm}$ for Image sensors. A possible explanation for the mentioned issues is that the Flexpoint sensor substrate might not be perfectly adherent to the hinge, especially for small rotation angles; in particular, a small initial bending (wrinkles) of the substrate could be present, thus affecting the initial response. On the other hand, friction between the hinge and the substrate should have little importance, since loading and unloading response curves are almost superimposed. Finally the GF factors were computed and a pretty constant value was detected for the Image sensors, while Flexpoint sensors showed an increasing behavior with bend angles.

\section{References}

[1] L. Dipietro, A.M. Sabatini, P. Dario "A survey of glovebased systems and their applications" IEEE Transaction on Systems, Man, and Cybernetics - part C: Applications and Reviews, vol.38, n.4, 2008, pp. 461482

[2] N.W. Williams "The Virtual Hand" Journal of Hand Surgery (British and European Volume), Vol. 22, No. 5, 560-567 (1997)

[3] N.W. Williams, J.M.T. Penrose, C.M. Caddy, E. Barnes, D.R. Hose, P. Harley "A goniometric glove for clinical hand assessment" Journal of Hand Surgery (British and European Volume, 2000) 25B: 2: 200-207

[4] L. Dipietro, A.M. Sabatini, P. Dario "Evaluation o fan instrumented glove for hand-movemnet acquisition" Journal of Rehabilitation Research and Development, vol.40, no.2, 2003, pp. 179-190

[5] T V Papakostas and N M White, 'Influence of substrate on the gauge factor of polymer thick-film resistors", $J$. Phys. D: Appl. Phys. 33 (2000) L73-L75 